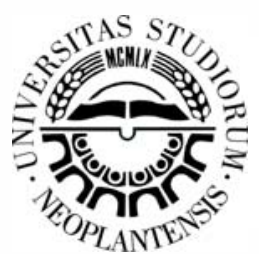

\title{
Influence of cutting parameters on the quality of the cut surfaces of steel swith a laser beam
}

\author{
Dobre Runchev ${ }^{a}$, Filip Zdraveski ${ }^{a}$, Irena Ivanova ${ }^{c}$ \\ ${ }^{a}$ University of Ss. Cyril and Methodius, Faculty of Mechanical Engineering - Skopje, Macedoniat \\ ${ }^{b}$ Bučim mine Radovis, Macedonia
}

\section{ABSTRACT}

The main objective of the research covered in this paper is to present results for the quality of surfaces thermally cut with a laser beam. The variety of steel materials used as samples on which laser cutting is performed are the following Č.0146 (1.0330), Č.0147 (1.0333), Č.2131 (1.5024), SS Ferbec CR, HARDOX 450 and HARDOX 550. Thermal cutting is carried out with a CNC controlled Fiber laser BAYKAL type BLS-F-1530. The quality of the cut surface is analyzed based on varying the power of the laser beam, changing cutting speed and the type of additional gas (oxygen, air and nitrogen). By visual inspection, measuring the roughness of the cut surface and measuring the width of the intersection, it is determined the influence of the factors like type of the base material, type of gases, the power of thelaser beam and the cutting speed, in accordance with the standards DIN EN ISO 9013-2002 and the JUS C.T3.022.

Key words: Laser cutting, cut surface, roughness measurement;

\section{INTRODUCTION}

The laser is an electro-optical device that converts electrical into electromagnetic energy, which further interacts with the material and turns into heat. The chamber (optical resonator) is the basic component of the laser Fig.1. Usually it is a mirror system, the first of which is completely reflective, and the last only partially reflective, ie. semi-permeable. The chamber is equipped with an active medium capable of a laser process. The active medium may be in solid, gaseous and very rarely in a liquid aggregate state. Accordingly, the lasers are divided into solid, gas and liquid $[1,2]$.

Fiber laser Fig. 2 and optical lasers are new technologies [3] of solid-state lasers that offer a combination of high quality light and wavelengths, which are easily absorbed by metal surfaces and are expected to counter $\mathrm{CO}_{2} \mathrm{Nd}$ : YAG laser in cutting metals of greater thickness (thickness greater than $2 \mathrm{~mm}$ ).

Fiber laser has high output power and an option for active regions long several kilometres and with very high optical gain.

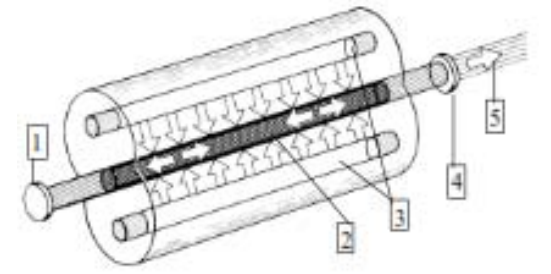

1-mirror

2-crystal 3-xenon lamp 4-partial mirror 5-laser beam

a) Solid laser (ND:YAG)

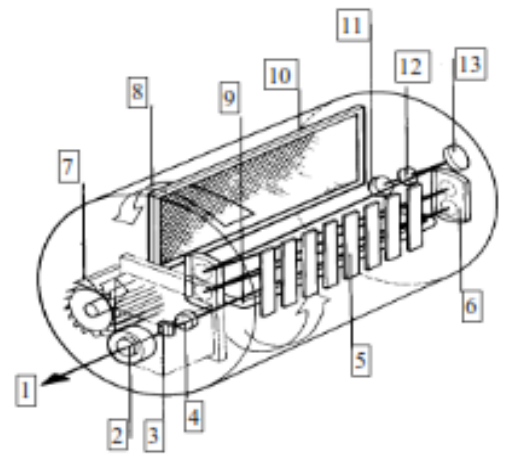

b) Cross flow Gas- $\mathrm{CO}_{2}$ laser

Fig.1 Chamber of laser 1-laser beam 2-output window 3,4-output coupler 5-segment anode 7-blower 8-gas direction 9-cathode 10-cooler 11-flowmeter 12-rear mirror 13-real time power monitor 6-folding mirror

\footnotetext{
*Corresponding author's.e-mail: dobre.runchev@mf.edu.mk,
} 
They have high-quality optical beam that allows fine cutting with a possible cut width less than $20 \mu \mathrm{m}$ $(0.02 \mathrm{~mm})$ on a thin section (foils) of metals with thickness $<200 \mu \mathrm{m}(0.2 \mathrm{~mm})$.

Cutting is with small characteristics and with very high accuracy and repeatability. Additional energy and better beam quality, provides clean cuts, reduction of edges and faster cutting.

Fiber laser light is channelled and is amplified through an optical cable similar to that used for data transmission. Light generation is about $200 \%$ more efficient than traditional $\mathrm{CO}_{2}$ lasers, where light transmission is much simpler, ie. without expensive optical mirrors, focussing on the lens and no moving parts.

This has a special advantage in terms of reducing maintenance requirements and operating costs.

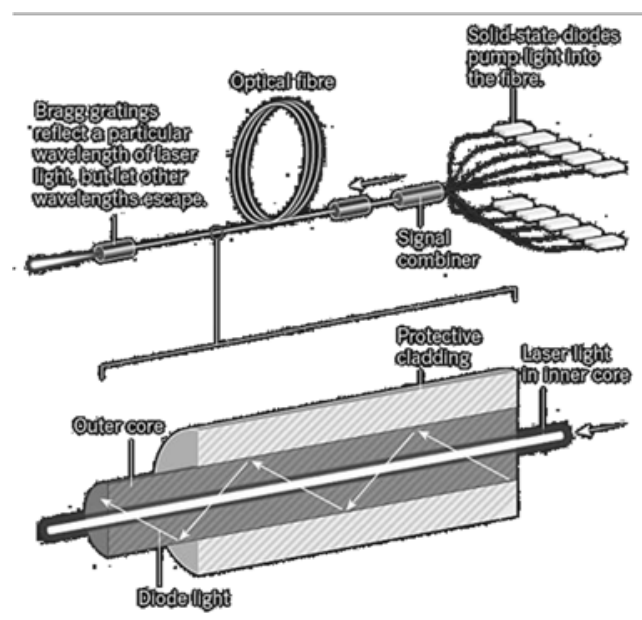

Fig.2 Fiber Laser principal scheme

Cutting of the samples for this research was done using a Fiber Laser (Fig.3) with double-cone nozzle, an auxiliary gas of $150 \mathrm{kPa}$.

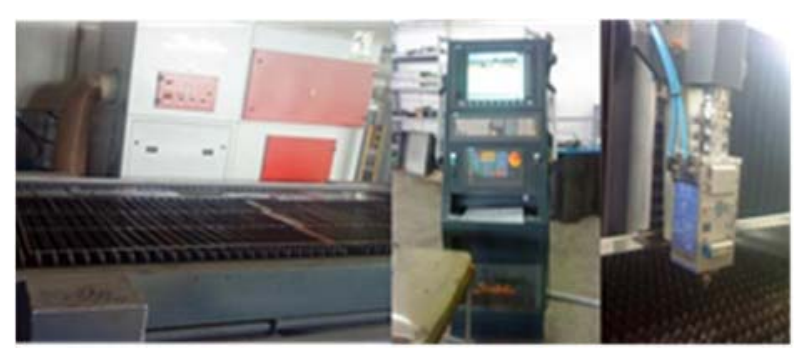

Fig.3 BAYKAL BLS-F-530

\section{THERMAL CUTTING PROCESS}

In thermal cutting with laser, cutting is done by burning, melting and evaporation of the material, depending on the type of cut material [4]. Thermal cutting is assisted by the kinetic energy of gases, which are used in cutting. Laser cutting is usually performed with coaxial gas delivery, high speed and pressure. The gas, depending on the nature of the materials being cut, can be: air, oxygen, hydrogen, argon or helium. The role of the gas is multistage, to intensify the cutting process, to improve the quality of the cut surface and to protect it from the surrounding influences and the like.

When cutting with burning, a pure oxygen supplement is used, and all types of steels, titanium, molybdenum and their alloys can be cut. When cutting with melting and evaporation, additional gas is used, hydrogen, and can be cut plastics, glass, wood, textiles and other non-metallic materials.

As a result of high thermal power, the laser is used for cutting various materials. Thus, with laser, thin sheets of high alloy steels, aluminum and copper can be cut with as well as non-metallic materials: ceramics, plastic, glass, wood, textile, paper, rubber and others.

With the laser can cut steel materials with thicknesses of 0.1 to $8 \mathrm{~mm}$. In the thinner elements of a structural carbon steel the intersection is narrower, so for a thickness up to $5 \mathrm{~mm}$ the intersection is $0.1 \mathrm{~mm}$ wide. When cutting this type of steel, oxygen is always used, that is, cutting by burning.

Stainless steels are cut at a lower speed and up to $50 \%$ compared to carbon steels. It can be cut with and without oxygen. When cutting with oxygen, oxides on the cut surface are possible [5].

The martensite and ferrite chromium alloy steels are cut with relatively high quality, compared with austenitic nickel alloyed steels that cut more difficult [6].

The quality of the laser cut-off surface depends on the nature of the cut material and the type of laser used, with its constant parameters determined by the type of active medium and optical system.

Cutting speed depends on:

- the type and thickness of the material to be cut;

- the focus position;

- the quality of oxygen;

- the density of energy;

- energy distribution after the cross-section of the laser.

\section{QUALITY OF CUT SURFACE}

The quality of the cut-off surface is measured and evaluated according to the roughness of the cut surfaces. The roughness of the surfaces in general terms represents the micro-geometric irregularity of the surface, which occurs during the cutting procedure. The roughness of the surfaces in certain cases significantly influences the working properties of the machine parts, especially in places where there is an interconnection of certain elements (friction, reaction, lubrication).

In general, the machined parts with a smaller roughness have greater dynamic strength, greater corrosion resistance, better heat transfer, and so on. To achieve a low degree of roughness is always associated with more and more expensive treatment technologies, which has the effect of increasing the cost of the part.

The value of roughness is usually measured as the mean reference line of the uneven profile $\mathrm{m}$, which divides the profile so that the measurement length 1 is the size of all deviations of the profile from that line to the smallest ones. Measurement length-height, depends on the type and 
quality of processing, as well as the measurement method. The roughness parameters are determined according to standard 4762, some of them according to the old standards JUS M.A1.020 and JUS M.A1.021 and DIN 4768.

For the estimation of the roughness of surfaces in practice, the mean arithmetic deviation of the $\mathrm{R}_{\mathrm{a}}$ profile is equal to the mean arithmetic value of the absolute value of the height of the profile for the measurement lenght $l$.

$R_{a}=\frac{1}{n} \sum_{1}^{n}\left|y_{i}\right|$

$R_{a}[\mu \mathrm{m}]-$ mean arithmetic deviation of the profile,

$l[\mu \mathrm{m}]-$ measured length,

$y_{x}, y_{i}[\mu \mathrm{m}]$ - the height of the roughness profile in relation to the mean reference line,

$n$ - number of points for estimating the height of the profile by length of measurement.
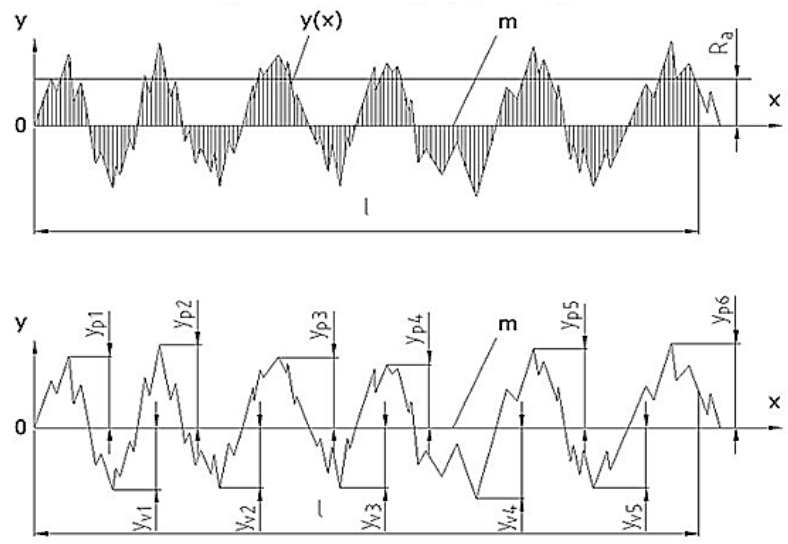

Fig.4Estimation of surface roughness

As a roughness parameter, medium height of roughness is often used $R_{z}$, which is equal to the sum of the arithmetic mean, to absolute values of the height of the five highest peaks and the arithmetic mean of the absolute values of the five largest depths, the depths of the measurement length $l$ (Fig.4).

$R_{Z}=\sqrt{\frac{1}{n} \sum_{1}^{n} y_{i^{2}}}$

$R_{Z}[\mu \mathrm{m}]$ - mean arithmetic deviation of the profile

$y_{i}[\mu \mathrm{m}]$ - height of the highest peak,

$n$ - number of points for estimating the height of the profile by length of measurement; at an approximate value of about $R_{z} \sim 4 R_{a}$

An important roughness parameter is the highest profile height $\mathrm{R} y$, which is defined as a distance between two directions parallel to the midline of the profile, drawn so that within the limits of the measuring length they touch the highest or the lowest point of the profile. This parameter is equal to the Rmax parameter (the largest uneven height), defined by the JUS standard, where the parameter is aproximatelly $\mathrm{R} \max =6.4 \mathrm{R} a$
According to the standard JUS M.A0.065 and DIN 4768, the ISO 1302 roughness of the technical surfaces is divided into 12 degrees, depending on the highest values of the mean arithmetical deviations $\mathrm{R} a$

\section{MeAsurement OF ROUGHNESS $\mathbf{R}_{z}$}

Measurements of the roughness of the cut pieces with a laser were performed with a measuring instrument: MITUTOYO SURFTEST SJ-400 (Fig.5).

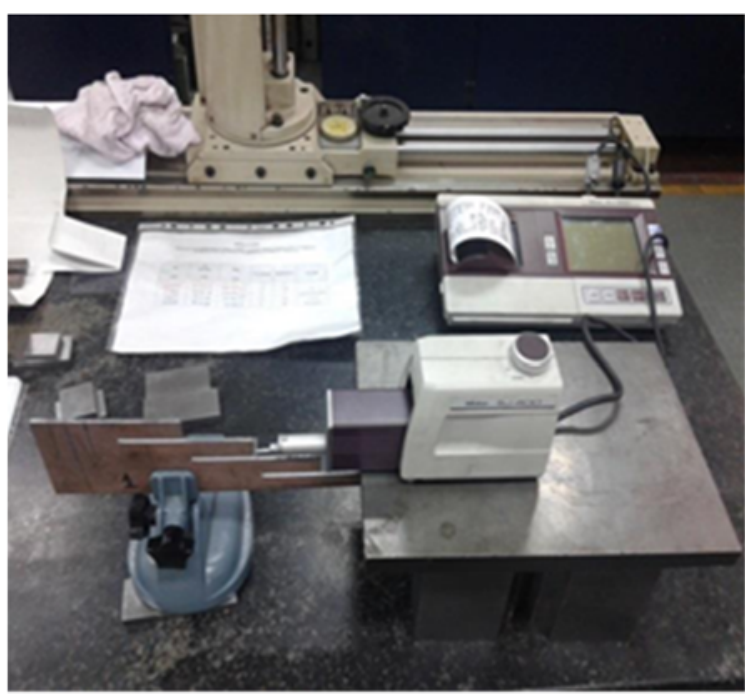

Fig 5 Measuring instrument

In accordance with [7] and [8] where the validity of the measuring tool is achieved by calibration using the C-type standard etalon, with a value of $R_{a}=2.94 \mu \mathrm{m}$ and $R_{\max }\left(R_{y}\right)$ of_9.3 $\mu \mathrm{m}$.

According to the recommendations contained in [9], the Gaussian filter of different size is used to obtain the roughness profiles from the primary profiles, depending on the size of the elementary measuring length.

The size of the perimeter $\mathrm{R}_{\mathrm{z} 5}$ is calculated in accordance with EN 9013. In the measurement, a reader without a slider with a radius of a measuring needle of $2 \mu \mathrm{m}$ was used.

\section{DETERMINATION OF QUALITY OF CUTS}

The results of measurements for the Hardox steel and C..0146 are given in the tables 1-4 and shown in Fig.6-13.

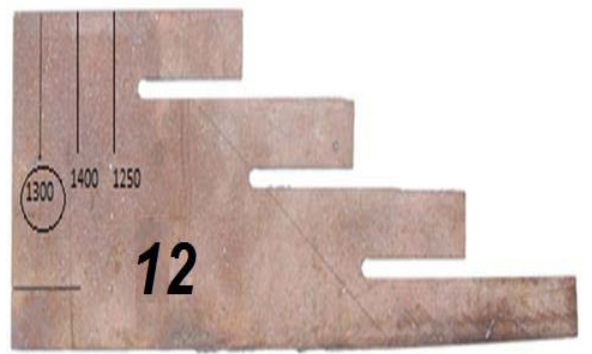

Fig. 6 Sample No. 12 Hardox 550 
Table 1 Cutting parameters sample 12

\begin{tabular}{|c|c|c|c|c|c|c|c|}
\hline z & 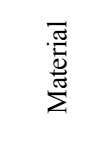 & 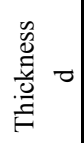 & 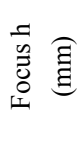 & 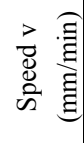 & 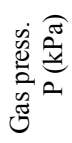 & $\begin{array}{l}\frac{z}{\tilde{N}} \\
\frac{\tilde{N}}{\tilde{N}} \\
\dot{z}\end{array}$ & 总 \\
\hline 1 & \multirow{3}{*}{$\begin{array}{c}\text { Hardox } \\
550\end{array}$} & \multirow{3}{*}{4} & \multirow{3}{*}{2.5} & 1250 & \multirow{3}{*}{85} & \multirow{3}{*}{1} & \multirow{3}{*}{$\mathrm{O}_{2}$} \\
\hline 2 & & & & 1400 & & & \\
\hline 3 & & & & 1300 & & & \\
\hline
\end{tabular}

Table 2 Measured roughness for sample 12

\begin{tabular}{|c|c|c|c|c|}
\hline $\begin{array}{c}\text { Vs } \\
(\mathrm{mm} / \mathrm{min})\end{array}$ & Sample & $R_{a}(\mu \mathrm{m})$ & $R_{z}(\mu \mathrm{m})$ & Range $R_{z 5}$ \\
\hline 1250 & 1 & 5.57 & 31.8 & \multirow{2}{*}{2} \\
\hline 1400 & 2 & 4.79 & 29.8 & \multirow{2}{*}{2} \\
\hline 1300 & 3 & 3.46 & 20.0 & \\
\cline { 1 - 4 } Mean & & 4.607 & 27.2 & \multirow{2}{|c|}{} \\
\hline
\end{tabular}

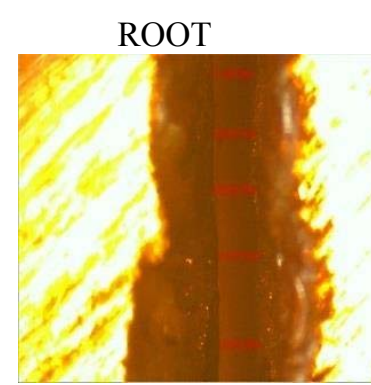

$\mathrm{b}_{\text {mean }}=0.252$

$\mathrm{v}=1300 \mathrm{~mm} / \mathrm{min}$

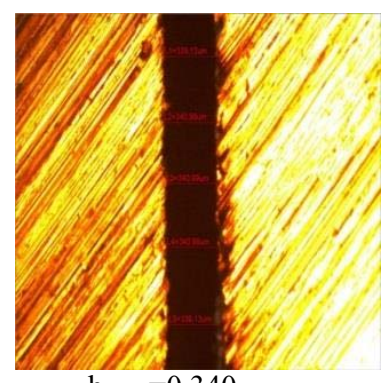

$\mathrm{b}_{\text {mean }}=0.340$

$\mathrm{v}=1400 \mathrm{~mm} / \mathrm{min}$

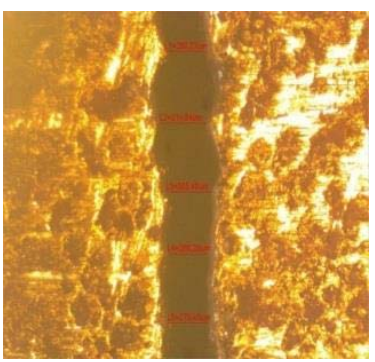

$\mathrm{b}_{\text {mean }}=0.289$

$\mathrm{v}=1250 \mathrm{~mm} / \mathrm{min}$
FACE

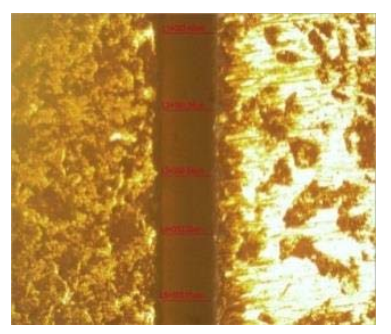

$\mathrm{b}_{\text {mean }}=0.354$

$\mathrm{v}=1300 \mathrm{~mm} / \mathrm{min}$
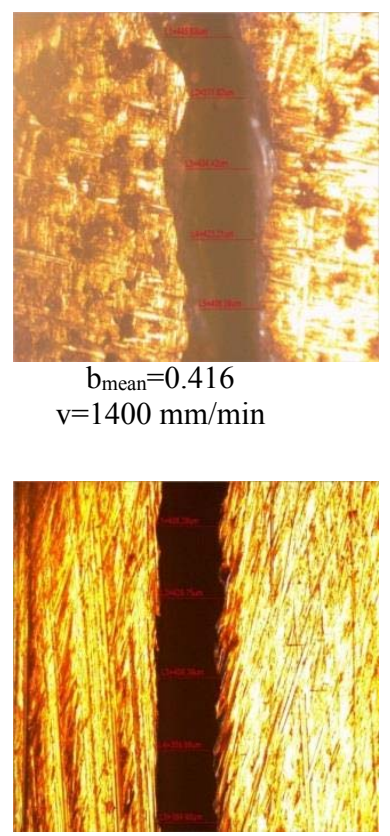

$\mathrm{b}_{\text {mean }}=0.397$

$\mathrm{v}=1250 \mathrm{~mm} / \mathrm{min}$
Fig.7 Average cross-section width sample 12

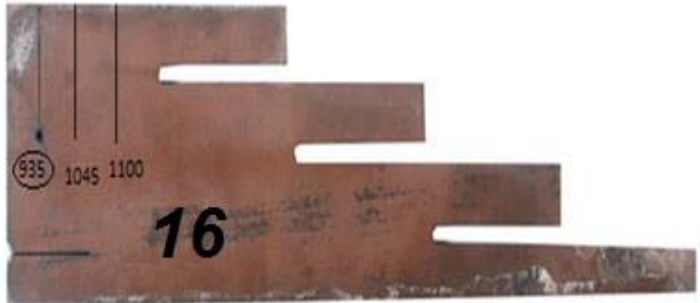

Fig. 8 Sample No. 16 Hardox 450

Table 3 Cutting parameters sample 16

\begin{tabular}{|c|c|c|c|c|c|c|c|}
\hline$\dot{\mathcal{Z}}$ & 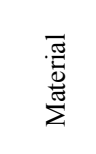 & $\begin{array}{l}\text { 己 } \\
\stackrel{\Xi}{\Xi} \\
\stackrel{\Xi}{\Xi}\end{array}$ & 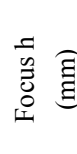 & $\begin{array}{l}> \\
\text { D } \\
\text { 园 } \\
\text { क }\end{array}$ & 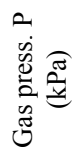 & $\begin{array}{l}\stackrel{0}{N} \\
\infty \\
\frac{0}{N} \\
0 \\
0 \\
Z\end{array}$ & : \\
\hline 1 & \multirow{3}{*}{$\begin{array}{c}\text { Hardox } \\
450\end{array}$} & \multirow{3}{*}{8} & \multirow{3}{*}{2.5} & 1100 & \multirow{3}{*}{105} & \multirow{3}{*}{1} & \multirow{3}{*}{$\mathrm{O}_{2}$} \\
\hline 2 & & & & 1045 & & & \\
\hline 3 & & & & 935 & & & \\
\hline
\end{tabular}
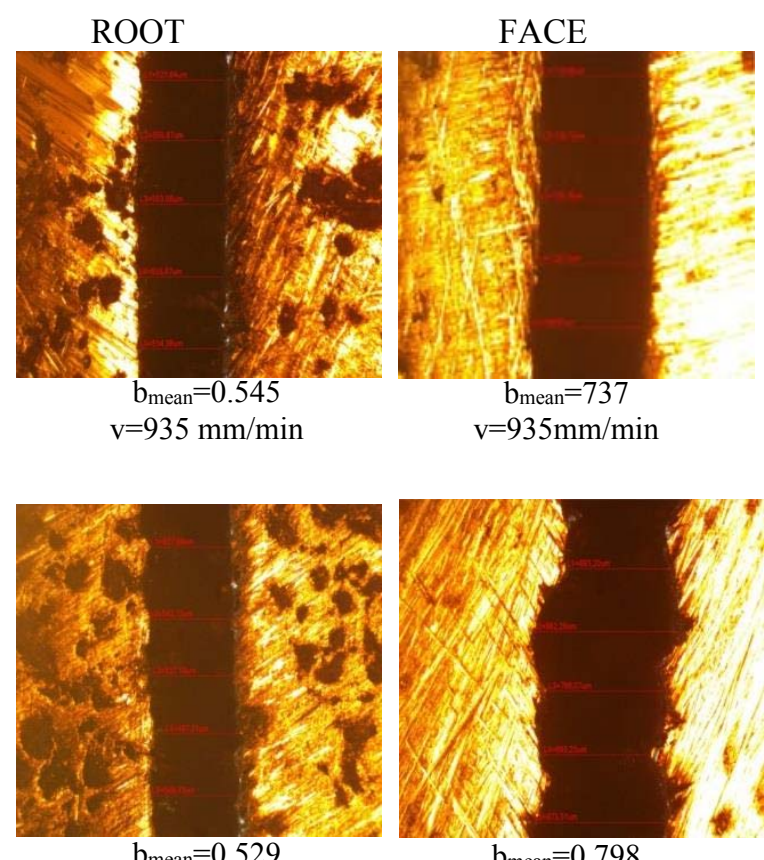

$=1045 \mathrm{~mm} / \mathrm{min}$

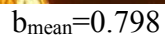

$\mathrm{v}=1045 \mathrm{~mm} / \mathrm{min}$

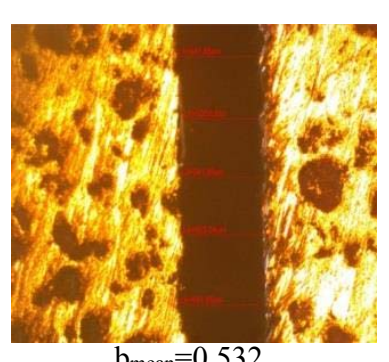

$\mathrm{v}=1100 \mathrm{~mm} / \mathrm{min}$ $\mathrm{b}_{\text {mean }}=0.532$

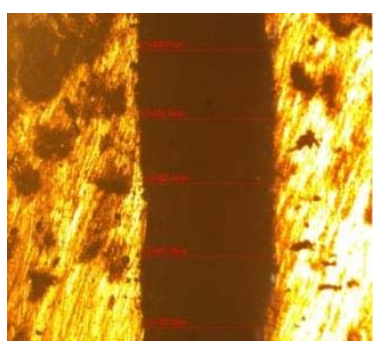

$b_{\text {mean }}=0.834$

$\mathrm{v}=1100 \mathrm{~mm} / \mathrm{min}$
Fig. 9 Average cross-section width sample 16 
Table 4 Measured roughness for sample 16

\begin{tabular}{|c|c|c|c|c|}
\hline $\begin{array}{c}\text { Vs } \\
(\mathrm{mm} / \mathrm{min})\end{array}$ & Sample & $R_{a}(\mu \mathrm{m})$ & $R_{z}(\mu \mathrm{m})$ & $\begin{array}{c}\text { Range } \\
0\end{array}$ \\
\hline 1100 & 1 & 10.9 & 48.8 & 3 \\
\hline 935 & 2 & 13.34 & 55.8 & 3 \\
\hline 1045 & 3 & 8.71 & 38.9 & 2 \\
\hline Mean & $\mathrm{x}$ & 10.65 & 47.83 & $\mathrm{x}$ \\
\hline
\end{tabular}

In the tables 5-8, visual control is presented, from photographs on cut surfaces of the same material Č.0146, but with different cutting speed and different setting of the height of the focus.

In Table 6, there is a big difference in the intersection.

The intersection of a piece marked as a test No. 4 has a smooth surface with sharp edges and straight line meshes, unlike sample No. 3, where there are uneven ends and the mesh lines are not properly arranged and are of varying depth.

Table 5 Visual control of specimen from Č.0146

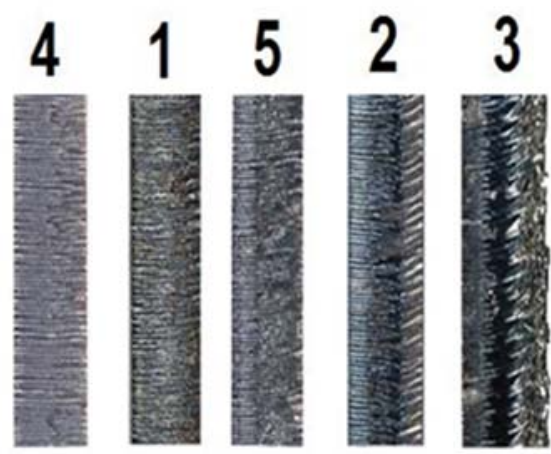

\begin{tabular}{|c|c|c|}
\hline \multicolumn{2}{|c|}{ Material } & Č.0146 \\
\hline \multicolumn{2}{|c|}{ Thickness } & 5 \\
\hline Specimen no. & Focus $\mathrm{h}[\mathrm{mm}]$ & Cutting speed \\
\hline 1 & 1.8 & \multirow{2}{*}{$\begin{array}{c}130 \\
\Omega\end{array}$} \\
\hline 5 & \multirow{4}{*}{3.3} & \\
\hline 4 & & 90 \\
\hline 2 & & 200 \\
\hline 3 & & 280 \\
\hline
\end{tabular}

Table 6 Results from roughness

\begin{tabular}{|c|c|c|l|c|c|}
\hline No. & $\begin{array}{c}\text { Speed } \\
{[\mathrm{mm} / \mathrm{min}]}\end{array}$ & $\begin{array}{l}\mathrm{R}_{\mathrm{Z} \min } \\
{[\mu \mathrm{m}]}\end{array}$ & $\begin{array}{l}\mathrm{R}_{\mathrm{Z} \text { mean }} \\
{[\mu \mathrm{m}]}\end{array}$ & $\begin{array}{l}\mathrm{R}_{\mathrm{Z} \max } \\
{[\mu \mathrm{m}]}\end{array}$ & $\begin{array}{c}\mathrm{R}_{\mathrm{z} 5} \\
(1-4)\end{array}$ \\
\hline 4 & 900 & 32.2 & 36.775 & 42.8 & 2 \\
\hline 5 & 1300 & 20.3 & 26.125 & 32.5 & 2 \\
\hline 2 & 2000 & 9.3 & 10.475 & 12.9 & 1 \\
\hline 3 & 2800 & 5.7 & 10.350 & 16.2 & 1 \\
\hline
\end{tabular}

Table 7. Cutting parameters sample 13 (Č.0146)

\begin{tabular}{|c|c|c|c|c|c|c|c|}
\hline$\dot{z}$ & 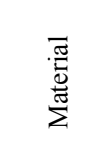 & 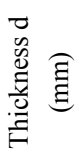 & $\begin{array}{l}\overrightarrow{0} \\
0 \\
0 \\
0\end{array}$ & 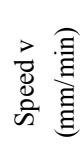 & 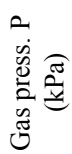 & 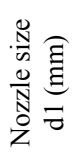 & 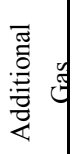 \\
\hline 1 & \multirow{3}{*}{ С̆.0146 } & \multirow{3}{*}{5} & \multirow{3}{*}{2.5} & 1300 & \multirow{3}{*}{$\begin{array}{l}9 \\
0\end{array}$} & \multirow{3}{*}{1} & \multirow{3}{*}{2} \\
\hline 2 & & & & 1500 & & & \\
\hline 3 & & & & 1600 & & & \\
\hline
\end{tabular}

Table 8. Measured roughness for sample 13

\begin{tabular}{|c|c|c|c|c|}
\hline $\begin{array}{c}\text { Vs } \\
(\mathrm{mm} / \mathrm{min})\end{array}$ & Sample & $R_{a}(\mu \mathrm{m})$ & $R_{z}(\mu \mathrm{m})$ & Range $R_{z 5}$ \\
\hline 1300 & 1 & 6.06 & 44.7 & \multirow{2}{*}{3} \\
\hline 1500 & 2 & 8.97 & 50.9 & \multirow{2}{*}{3} \\
\hline 1600 & 3 & 9.71 & 57.2 & \\
\hline Mean & & 8.247 & 50.93 & \\
\hline
\end{tabular}

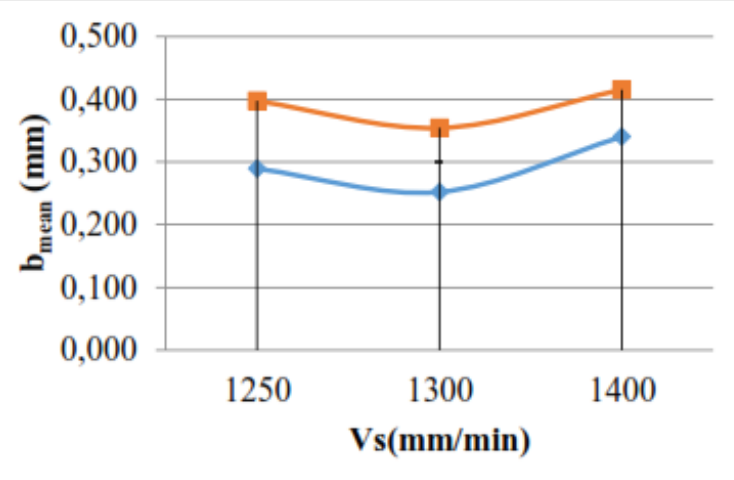

Fig. 10 Curve speed- $b_{\text {mean }}$ for sample 12

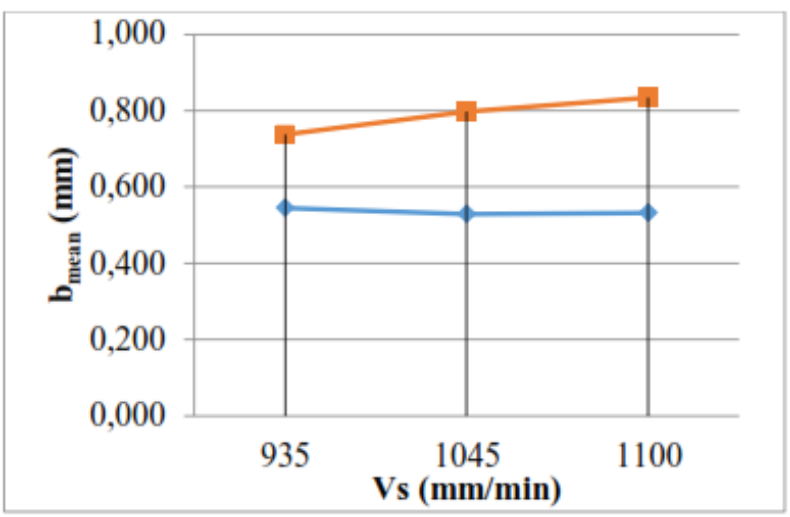

Fig. 11 Curve speed- $b_{\text {mean }}$ for sample 16

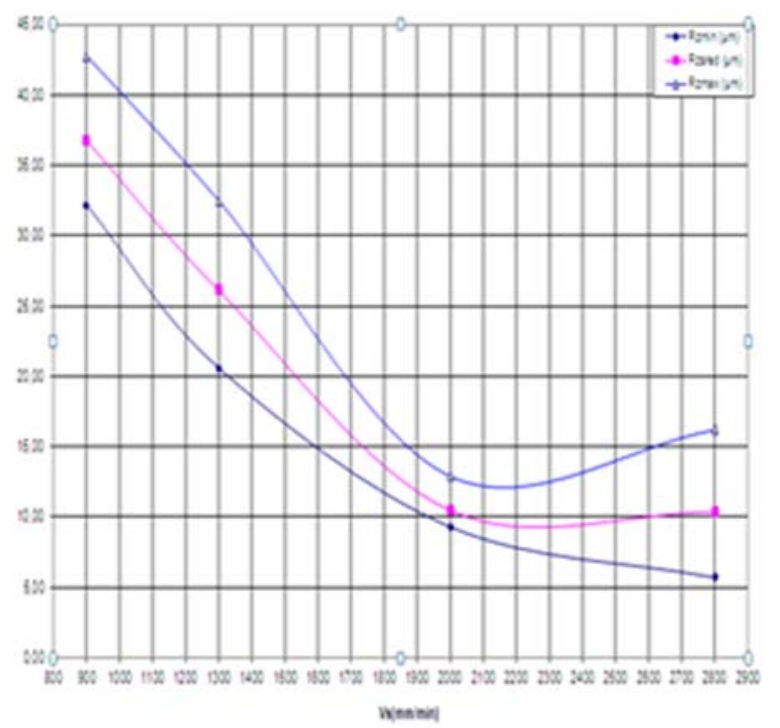

Fig. 12 Diagram for measured roughness $f(v)$ 


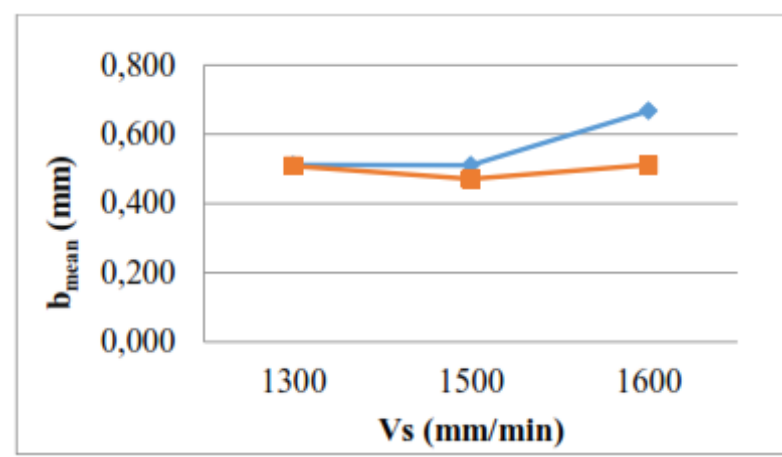

Fig. 13 Curve speed-b $b_{\text {mean }}$ for sample 13

\section{CONCLUSION}

Based on analysis of the experimental testing of structural steels such as Č.0146 and alloy steels with increased strength and hardness HARDOX 450 and HARDOX 550 conclusions can be made concerning quality of laser cutted surface. The cutted surface quality depends from different factors such as:

- type and thickness of the material on which the intersection is performed,

- cutting speed,

- the position of the focus,

- laser mode (distribution of energy per cross section of the laser),

- the energy density,

- the quality of oxygen.

The width and parallelity of the intersection should be aligned with the roughness class on the intersection surfaces simultaneously.

After the completion of thermal laser cutting of structural steel C. 0146 , better visual section is seen on the piece on which the intersection takes place at a lower speed, unlike section made with greater speed.

According to the visual control and according to the calculations of $b_{\text {mean }}$ of the Hardox steels, a good quality cross section is obtained. To improve the intersection quality, cutting needs to be executed with greater speed.

\section{REFERENCES}

[1] J.Powell: CO2 Laser Cutting, Springer Verlag, 1993.

[2] M.Faerber, W.Schmidt: Laserschneidgase, DVSBerichte Band 185, DVS-Verlag, S.72-74, 1997.

[3] H.Zellmer u.a.: Fiber lasers - Novel light sources in research and industry, Fraunhofer IOF Jahresbericht 2004, pp. 39-41, 2004.

[4] H.Mair: Thermische Schneidverfahren, Sonderdruck 128, DVS Berichte, Band 109, S.4-8, 1987.

[5] A. Amulevicius, K.Mazeika, C. Sipavicius, Oxidation of SS by laser cutting, Acta Physica Polonica A, vol.115 (5), S.880-885, 2009.

[6] A. Parhiban et al.,Optimisation of $\mathrm{CO} 2$ laser cutting parameters on Austenitic type Stainless steel sheet, IOP conf. series: Materials Science and Engineering, vol. 183,S.12-22, 2017.

[7] N.N: Geometrical product specifications (GPS) Surface texture: Profil method - Calibration of contact (stylus) instruments, EN ISO12179, 2000.

[8] N.N: Geometrical product specifications (GPS) Surface texture: Profil method - Calibration of contact (stylus) instruments, EN ISO12179, 2000.

[9] N.N: Geometrical product specifications (GPS) Surface texture: Profil method - Rules and procedures for the assessment of surface texture. 